\title{
Formulation and Evaluation of a New Biodegradable Periodontal Chip Containing Thymoquinone in a Chitosan Base for the Management of Chronic Periodontitis
}

\author{
Fouad H. Al-Bayaty, ${ }^{1}$ Azwin A. Kamaruddin, ${ }^{1}$ Mohd. A. Ismail, ${ }^{1}$ and Mahmood A. Abdulla ${ }^{2}$ \\ ${ }^{1}$ Center of Study for Periodontology, Faculty of Dentistry, Universiti Teknologi MARA (UiTM), 40450 Shah Alam, \\ Selangor, Malaysia \\ ${ }^{2}$ Department of Molecular Medicine, Faculty of Medicine, University of Malaya, 50603 Kuala Lumpur, Malaysia
}

Correspondence should be addressed to Fouad H. Al-Bayaty; drfouadhm@yahoo.com

Received 13 January 2013; Revised 10 March 2013; Accepted 10 March 2013

Academic Editor: Abdelwahab Omri

Copyright (C) 2013 Fouad H. Al-Bayaty et al. This is an open access article distributed under the Creative Commons Attribution License, which permits unrestricted use, distribution, and reproduction in any medium, provided the original work is properly cited.

\begin{abstract}
Objective. This study was performed to develop a biodegradable periodontal chip containing thymoquinone and to evaluate its effectiveness for managing chronic periodontitis. Methods. Chips were formulated from thymoquinone and chitosan. Twelve patients with periodontal pockets measuring $\geq 5 \mathrm{~mm}$ participated in this study. Overall, 180 periodontal pockets were evaluated. At day zero, all patients were treated with full-mouth scaling and root planning. Periodontal pockets were divided into three groups. Group one served as the control group, while group two received plain chitosan chips. Group three received chips containing thymoquinone. Plaque index, bleeding upon probing, periodontal probing pocket depths, and clinical attachment levels were recorded at days 0 and 60. Results. The statistical significance of differences was tested with a paired sample $t$-test, a Chi-squared test, and a one-way ANOVA. The results indicated significant improvement in plaque index and bleeding upon probing and a reduction in periodontal pockets from baseline in all four groups $(P<0.05)$. Gains in clinical attachment levels were significantly higher $(P<0.005)$ in the group receiving thymoquinone chips compared to other groups. Conclusion. Periodontal chips containing thymoquinone can be used as adjuncts for the treatment of patients with chronic periodontitis.
\end{abstract}

\section{Introduction}

Periodontitis is an inflammation of the periodontium that extends beyond the gingiva and destructs the connective tissue to which teeth attach. Bacteria associated with dental plaque have been widely accepted as the cause of inflammatory periodontal diseases [1]. Traditionally, periodontal therapy consists of changing the periodontal environment to a one that is unfavorable for the preservation of bacterial plaque in areas of gingival tissues. To realize this goal, therapeutic regimes comprise procedures such as oral health instruction to attain satisfactory oral hygiene, scaling, rectification of improper restorative dentistry, root planning, and the elimination of pockets or anatomical defects by surgical means. However, with increases in our knowledge and understanding of the bacterial aetiology of periodontal disease, and particularly the introduction of the theory that certain bacteria are included in periodontal disease [2], a more direct method using antibacterial agents has become an important aspect of periodontal remedial regimes. In addition, the treatment of chronic periodontitis focuses on arresting the destruction of periodontal support through the removal of some pathogenic bacteria present in the periodontal pocket. Mechanical scaling and root planning accomplish this goal. Nevertheless, the presence of tortuous and deep pockets often results in considerable variation in the effectiveness of scaling and root planning, which is one reason to employ antimicrobial agents in the management of chronic periodontitis [3]. These antimicrobial agents can be delivered to diseased sites by systemic or local administration [4]. The periodontal pockets provide natural liquid containment immersed in gingival crevicular fluid that allows easy 
access for delivery equipment. This fluid furnishes a filtering medium for a drug to be released from a solid dosage structure and for its dispersion throughout the pocket. These factors make the periodontal pocket a natural site for the introduction of medication with a local sustained-release delivery system. PerioChip, a pioneer in the field of biodegradable delivery frameworks for diminishing pocket depth in chronic periodontitis, is devised as an adjunctive therapy to scaling and root planning. PerioChip contains chlorhexidine gluconate, an antiseptic agent. PerioChip is a small, orangetan, rectangular chip (rounded at one end) to be inserted into periodontal pockets. Each PerioChip weighs approximately $7.4 \mathrm{mg}$ and holds $2.5 \mathrm{mg}$ of chlorhexidine gluconate in a biodegradable matrix of hydrolyzed gelatin (cross-linked with glutaraldehyde). Many studies have shown the clinical effectiveness of dental professionals for the long-term management of adult periodontitis $[5,6]$. However, the usage of PerioChip has shown some adverse effects in previous trials. Brown discoloration of teeth, restorative materials, a bitter taste; and occasional sloughing of oral mucosa have been reported when chlorhexidine is used as mouth rinses [1]. To overcome these adverse effects, the World Health Organization (WHO) advocates that researchers investigate the possibility of using natural products, such as plant extracts and herbs, as alternatives [7]. Plant extracts and herbs that have been used previously include Salvadora persica [7], Arnica montana, and Hamamelis virginiana which have been shown to exhibit antibacterial activity against periodontopathic bacteria $[8,9]$. Nigella sativa (NS), commonly known as black seed or black cumin, is an annual plant belonging to the Ranunculaceaefamily. The herb has been traditionally used in the Indian subcontinent, Arabian countries and Europe for culinary and medicinal purposes. Nigella sativa seeds contain diverse but well-characterized chemical components, many of which are capable of inducing beneficial pharmacological effects in humans [10]. Nigella sativa includes essential oils, proteins, alkaloids and saponins [11]. Most of the properties of Nigella sativa or its extracts are attributed to thymoquinone (2-isopropyl-5-methyl-1, 4-benzoquinone, TQ), which is one of the monoterpenoid hydrocarbon compounds of Nigella sativa's volatile oil [12] states. Thymoquinone exhibits inhibitory and bactericidal effects against both Gram-positive and Gram-negative bacteria, and even shows synergistic effects when combined with other antibiotics. Other studies have shown that thymoquinonehas various therapeutic effects, such as acting as a diuretic and hypotensive, attacking tumor cells, antihistamine, anti-inflammatory and analgesic properties, and antiepileptic effects. Moreover, thymoquinone exhibits chemical (nonenzymatic) metabolic activity dependent on GSH, NADPH, or NADH that may represent a "cellular switch" capable of modulating cellular antioxidant defenses [13]. Chitosan has gained attention as a drug delivery carrier because it is a stable, biodegradable, nontoxic hydrophilic polysaccharide with phenomenal mucoadhesive properties and permeation improving factors [14]. No studies have been performed to develop a periodontal chip containing thymoquinone for the treatment of chronic periodontitis. Therefore, the aim of this study is to develop periodontal chip containing thymoquinone and to evaluate the effectiveness of a biodegradable periodontal chip with thymoquinone in a chitosan base as a targeted drug conveyance in the management of chronic periodontitis.

\section{Materials and Methods}

\subsection{Experimental Subjects}

2.1.1. Study Design. A two-month randomized single-blind split mouth study was completed in the Faculty of Dentistry of the Universiti Teknologi MARA Shah, Alam, Malaysia. The methodology used was affirmed by the institutional committee of ethics on human research of the Universiti Teknologi MARA 600-RMI (5/1/6/01), where all the participants signed a written informed consent agreeing on their cooperation in the research project. This study was divided into two parts: laboratory procedures and the clinical trial. Laboratory procedures involved the preparation of periodontal chips and clinical trial involved periodontal therapy and chip insertions.

2.1.2. Study Populations. Twelve male patients with chronic periodontitis aged between 35 and 56 years (mean age $41.8 \pm$ 5.6) with at least four nonadjacent teeth with periodontal pockets measuring $\geq 5 \mathrm{~mm}$ were chosen to participate in this randomized split mouth study. The patients were recruited from amongst patients' referred to the Center of Study for Periodontology, Faculty of Dentistry, Universiti Teknologi MARA. At the screening visit, orthopantomograph X-rays were taken to confirm the diagnosis, and the relevant pockets were identified and recorded on patient charts. From these twelve patients, 1656 periodontal pockets were examined; however, only 180 periodontal pockets measuring $\geq 5 \mathrm{~mm}$ which bled when probed were detected and evaluated. Patient exclusion criteria included a history of systemic disease that might impact the course of periodontal disease or would require prophylactic antibiotics before dental treatment; antibiotic usage or any modality of periodontal treatment occurring in the past three months; the presence of overhanging restorations, pregnancy, smoking habits; or allergy to Nigella sativa and/or thymoquinone.

\subsection{Laboratory Procedures}

2.2.1. Preparation of the Biodegradable Chitosan Chip. To prepare the biodegradable chitosan chip, $1 \%$ acetic acid was added into $5 \mathrm{gm}$ of chitosan powder (purchased from Sigma, Germany) and incubated overnight. This mixture was then dissolved in water and sonicated to form a homogenous combination, and poured into uniquely designed rectangular glass molds lined with aluminum foil. After drying overnight at room temperature, the resultant film was cut into small rectangular chips $0.5 \times 0.5 \mathrm{sq} \cdot \mathrm{cm}$ in size and $0.16 \pm 0.02 \mathrm{~mm}$ thick. These chips were then covered in aluminum foil and kept in sterile vials at room temperature [15].

2.2.2. Preparation of Thymoquinone Chips Containing Biodegradable Chitosan. Thymoquinone (purchased from Sigma, 
USA) $(0.25 \mathrm{mg} ; 100 \% \mathrm{w} / \mathrm{w})$ was dissolved and added to chitosan that had been soaked in $1 \%$ acetic acid overnight. Both ingredients were sonicated to form a homogenous mixture and poured into a uniquely designed rectangular glass mold lined with aluminum foil. After drying overnight at room temperature, the resultant film was cut into small rectangular chips $0.5 \times 0.5 \mathrm{sq} \cdot \mathrm{cm}$ in size. A content uniformity test was conducted on some chips in a random fashion to affirm the quantity of drug administered in every chip. The chips were then stored in sterile vials and kept at room temperature.

2.3. Clinical Trial. Before commencement of the clinical trial, the patients were given subject information sheets to explain the research procedures in detail and a consent form was signed by each patient. An alginate impression was taken of both arches and a soft transparent acrylic stent was constructed. This acrylic stent was used as an aid in localizing the exact site of measurement to ensure that the same site was being measured at each visit. Initially, examinations that included a thorough evaluation of the periodontal condition of the patients' teeth were performed. All patients received full-mouth supragingival scaling and polishing and were instructed to practice a standard effective oral hygiene routine consisting of subgingival brushing. All clinical measurements were performed by a single examiner (Mohd. A. Ismail) who was unaware of the treatments performed upon each subject. The 180 periodontal pockets were randomized in three groups prior to baseline.

The following clinical parameters were recorded at day 0 and day 60 following treatment: plaque index (PLI), bleeding on probing (BOP), clinical probing pocket depth (PPD) and clinical attachment level (CAL). Probing pocket depths were measured using a UNC probe. Plaque and BOP were categorized as absences or presences (on a 0/1 scale). BOP was considered present if bleeding occurred within $20 \mathrm{~s}$ after pocket probing [16]. The clinical attachment level (CAL) was evaluated using a customized acrylic stent

After recording baseline measurements, all study pockets were root planed under local anesthesia with Gracey curettes (Hu-Friedy, Chicago, IL, USA), a procedure that was conducted by one investigator (Azwin A. Kamaruddin). Moreover, a single chip was placed into each of the designated periodontal pockets after SRP, in groups 2 and 3 using dental forceps, after isolating and drying the associated tooth. The tips of the forceps, or a dental probe, were then used to maneuver the chip into position until it was totally submerged apical to the gingival margin. Chips were applied subgingivally.

Group 1. It consisted of 60 sites, received scaling and root planning alone (control group).

Group 2. It consisted of 60 sites, received scaling and root planning with chitosan chip insertion.

Group 3. It consisted of 60 sites, received scaling and root planning followed by thymoquinone chip insertion.

Patients were examined two days following the placement of chips for assessment. Patients were instructed not to use dental floss for 10 days to avoid displacement of the chip and to avoid any types of mouth rinses or oral irrigation devices during the study period. At day 14, patients were called back for second chip insertions and the measurement of the plaque index and the presence of bleeding on probing were checked. On the last day of the clinical trial (day 60), all of the clinical parameters measured were recorded again.

2.3.1. Intraexaminer Agreement. Intraexaminer calibration was performed on measurements of plaque index (PLI), bleeding on probing (BOP), and periodontal pocket depth (PPD). A total of 180 sites were measured on each patient and the records were charted. Then, 30 minutes after the first measurement, the examiner remeasured the same 180 sites. These measurements were repeated twice on every patient. Data were inserted into SPSS and Cohen's kappa coefficient was calculated. The result of the analysis was kappa $=0.81$ $(P<0.001)$, which shows almost perfect agreement.

\section{Results}

There were no patient withdrawals over the study period.

3.1. Mean Plaque Index after and before Treatment. All three groups showed a reduction of and improvement in sites with visible supragingival plaque index after and before treatment. The thymoquinone and chitosan chip groups showed significant improvement in plaque index after treatment. However, for the control group, there was no significant difference in plaque index between after and before treatment measurements $(P<0.05$ (Table 1$))$.

3.2. Mean Bleeding on Probing after and before Treatment. There was significant improvement in the presence of bleeding on probing in all groups after treatment. The enhancements in BOP were comparatively similar for all groups after the two months of the study $(P=0.05$ (Table 2$)$ ).

3.3. Mean Periodontal Pocket Depth after and before Treatment. The mean periodontal pocket depth was measured after and before treatment. The results showed reductions in PPD in all three groups, and these reductions are statistically significant in all three groups $(P=0.01)$. Table 2 shows the mean changes in PPD after and before treatment. All groups had significant changes in PPD after the two months of the study $(P=0.01)$. The effects of the treatments were evident in the posttreatment recording. At two months, PPD was reduced to $4.52 \mathrm{~mm}$ in the SRP alone group, to 5.27 in the SRP plus chitosan group, and to $4.83 \mathrm{~mm}$ in the SRP plus thymoquinone chip group, contrasted with pretreatment records. The total mean reductions in PPD were $0.82 \mathrm{~mm}$ for the SRP alone group, $1 \mathrm{~mm}$ for SRP plus chitosan group, and $1.33 \mathrm{~mm}$ for the thymoquinone chip group. The improvements in PPD were more evident in the groups treated with SRP and the Thymoquinone chip (Table 3 ).

3.4. Clinical Attachment Level. The results indicated increases in the clinical attachment levels in each of the three 
TABLE 1: Mean differences in the plaque index between groups preand post-treatment.

\begin{tabular}{lcccc}
\hline Type of treatment & $N$ & Pearson Chi-Squared test & df & Significance \\
\hline Control & 60 & 0.292 & 1 & NS \\
Chitosan & 60 & 0.031 & 1 & $\mathrm{~S}^{*}$ \\
Thymoquinone & 60 & 0.036 & 1 & $\mathrm{~S}^{*}$ \\
\hline
\end{tabular}

${ }^{*}$ Significant at the $(P<0.05)$ level.

TABLE 2: Mean bleeding on probing pre- and post-treatment.

\begin{tabular}{lcccc}
\hline Type of treatment & $N$ & Pearson Chi-Squared test & df & Significance \\
\hline Control & 60 & 0.292 & 1 & NS \\
Chitosan & 60 & 0.031 & 1 & $\mathrm{~S}^{*}$ \\
Thymoquinone & 60 & 0.036 & 1 & $\mathrm{~S}^{*}$ \\
\hline
\end{tabular}

${ }^{*}$ Significant at the $(P<0.05)$ level.

TABLE 3: Mean periodontal pocket depth pre- and post-treatment.

\begin{tabular}{|c|c|c|c|c|}
\hline $\begin{array}{l}\text { Type of } \\
\text { Treatment }\end{array}$ & Visits & $\begin{array}{c}\text { Pearson } \\
\text { Chi-Squared } \\
\text { Test }\end{array}$ & df & Significance \\
\hline Control & $\begin{array}{l}\text { Pre-treatment } \\
\text { Post-treatment }\end{array}$ & 0.024 & 1 & $S^{*}$ \\
\hline Chitosan & $\begin{array}{l}\text { Pre-treatment } \\
\text { Post-treatment }\end{array}$ & 0.008 & 1 & $S^{*}$ \\
\hline Thymoquinone & $\begin{array}{l}\text { Pre-treatment } \\
\text { Post-treatment }\end{array}$ & 0.023 & 1 & $S^{*}$ \\
\hline
\end{tabular}

${ }^{*}$ Significant at the $(P<0.05)$ level.

groups. The thymoquinone group showed significantly greater improvement than the other groups, with the highest measured gain of $1.33 \mathrm{~mm}$, while the chitosan group showed an average gain in CAL of $1.00 \mathrm{~mm}$ and the control group had an average $0.82 \mathrm{~mm}$ gain (Figure 1 ).

\section{Discussion}

Nigella sativa has been used previously in few clinical trials. In one trial, NS oil extract administered at a dose of $40 \mathrm{mg} / \mathrm{kg}$ significantly improved clinical symptoms in patients with allergic diseases such as bronchial asthma, allergic rhinitis, and atopic eczema [17]. Adverse effects did not occur; the tolerability of NS oil was further confirmed in a separate study [5], and the crude drug did not produce any adverse side effects [18]. Thymoquinone is considered to be the main active ingredient in NS oil [19]. It represents $18.4 \%$ to $24 \%$ of the $N$. sativa volatile oil [20]. Pharmacological investigations of NS seed extracts reveal a broad spectrum of activities including antioxidant [21], anti-inflammatory [13] and antimicrobial [22] activities. In the current study, the effects of the treatments were evident in all groups, but the reduction in PPD obtained in the SRP plus thymoquinone chip group was higher than that obtained in the other groups. The PPD reduction in this group $(1.33 \mathrm{~mm})$ was more significant than what has been observed in other studies performed by $[4,23]$ using SRP plus a CHX chip. These studies reported mean PD

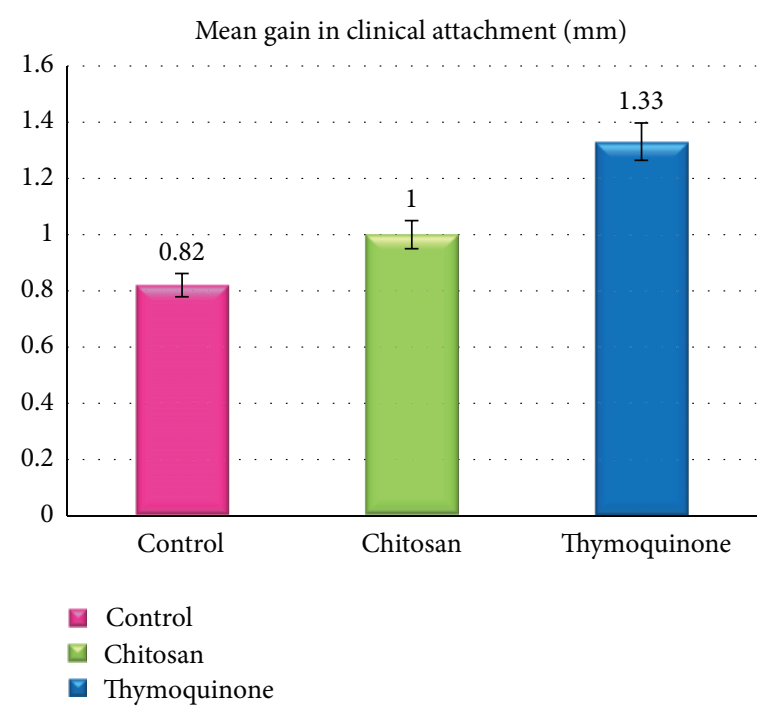

FIgURE 1: Mean gain in clinical attachment in all groups. All values are expressed as the means; the mean difference is significant at the $(P<0.05)$ level. Data were analyzed using a paired sample $t$-test, a Chi-squared test, and a one-way ANOVA.

decreases of $0.84 \mathrm{~mm}$ and $0.80 \mathrm{~mm}$, respectively. Our results are also in agreement with studies performed by $[6,15,24]$ using periodontal chips containing chlorhexidine as local delivery agents in the management of chronic periodontitis. These previous studies demonstrated that when a biodegradable chlorhexidine chip is used as an adjunct to conventional periodontal therapy critical enhancements are obtained in periodontal probing depths and attachment levels compared to the use of scaling and root planning alone. In addition, researchers have found that the clinical signs of periodontitis also significantly improved when periodontal chips were used as adjuncts compared to scaling and root planning alone. In the present study, the use of thymoquinone chips resulted in a dramatic increase in clinical attachment levels compared to a control group and chitosan chip usage. This could be a result of the combined impact of the antimicrobial effect of thymoquinone and chitosan or the synergistic effects of the components. We attempted to use the same concentration $(2.5 \mathrm{mg}$ ) of chitosan used in CHX chips, while $0.25 \mathrm{mg}$ of the active component of thymoquinone was used in the thymoquinone chips. We believe that increasing the concentration of thymoquinone in the chips may lead to better results. It is critical to recognize the improvement of gingival inflammation throughout the study presented by the significant changes in BOP before and after treatment in all groups. These findings were in agreement with several previous studies $[15,24]$. Significant changes in visible plaque were found before and after treatment in all treatment groups receiving chips (and not in the control group or the group that received SRP alone). One of the limitations of this study is that we could not compare our chip with CHX chips as CHX chips are very expensive. Further studies are necessary to test our chips in longer clinical trials, to increase the concentration of thymoquinone in the chips, and to compare 
thymoquinone chips with the commercially available $\mathrm{CHX}$ chips. Periodontal chips containing thymoquinone can be used at the same appointment as scaling and root planning or during periodontal maintenance appointments. In view of this research, periodontal chips formulated from thymoquinone incorporated in a chitosan base as a targeted method of drug delivery provide clinical benefits that are achieved when using these chips as an adjunct to conventional scaling and root planning in the management of chronic periodontitis patients.

4.1. Statistical Analyses. Mean values per patient of the clinical parameters were calculated for every treatment group at examination. Updates to the clinical parameters were computed for each site in test and control groups. Updates to PLI, BOP, PPD, and clinical attachment level (CAL) between baseline and day 60 were analyzed among the treatment groups. All data were gathered and analyzed using Statistical Package for Social Science (SPSS) version 20. The statistical significance of differences was tested with a paired sample $t$-test, a chi-squared test, and a one-way ANOVA. Significance was accepted at the probability level $P<0.05$.

\section{Acknowledgments}

The authors express gratitude to the Universiti Teknologi MARA for financial support Grant no. 600-RMI/ST/ DANA5/3/Dst (198/2009). The authors would like to thank the University of Malaya for funding this research. Grant no. HIR- MOHE (F000009-21001).

\section{References}

[1] G. C. Armitage, "Periodontal diagnoses and classification of periodontal diseases," Periodontology 2000, vol. 34, pp. 9-21, 2004.

[2] P. D. Marsh, A. Moter, and D. A. Devine, "Dental plaque biofilms: communities, conflict and control," Periodontology 2000, vol. 55, no. 1, pp. 16-35, 2011.

[3] A. H. Vinholis, L. C. Figueiredo, E. M. Júnior, R. A. Marcantonio, S. L. Salvador, and G. Goissis, "Subgingival utilization of a $1 \%$ chlorhexidine collagen gel for the treatment of periodontal pockets. A clinical and microbiological study," Brazilian Dental Journal, vol. 12, no. 3, pp. 209-213, 2001.

[4] D. Dodwad, S. Vaish, A. Mahajan, and M. Chhokra, "Local drug delivery in periodontics: a strategic intervention," International Journal of Pharmacy and Pharmaceutical Sciences, vol. 4, no. 4, pp. 30-34, 2012.

[5] A. Mombelli, N. Cionca, and A. Almaghlouth, "Does adjunctive antimicrobial therapy reduce the perceived need for periodontal surgery?" Periodontology 2000, vol. 55, no. 1, pp. 205-216, 2011.

[6] P. A. Heasman, L. Heasman, F. Stacey, and G. I. McCracken, "Local delivery of chlorhexidine gluconate (PerioChip) in periodontal maintenance patients," Journal of Clinical Periodontology, vol. 28, no. 1, pp. 90-95, 2001.

[7] F. H. Al-Bayaty, A. H. AIKoubaisi, N. A. W. Ali, and M. A. Abdulla, "Effect of mouth wash extracted from Salvadora persica (Miswak) on dental plaque formation: a clinical trial," Journal of Medicinal Plants Research, vol. 4, pp. 1446-1454, 2010.
[8] S. Vahabi, E. Najafi, and S. Alizadeh, "In vitro antimicrobial effects of some herbal essences against oral pathogens," Journal of Medicinal Plants Research, vol. 5, pp. 4870-4878, 2011.

[9] L. Iauk, A. M. Lo Bue, I. Milazzo, A. Rapisarda, and G. Blandino, "Antibacterial activity of medicinal plant extracts against periodontopathic bacteria," Phytotherapy Research, vol. 17, no. 6, pp. 599-604, 2003.

[10] M. A. Randhawa and M. S. Al-Ghamdi, "A review of the pharmacotherapeutic effects of Nigella sativa," Pakistan Journal of Medical Research, vol. 41, pp. 77-83, 2002.

[11] N. Ismail, M. Ismail, A. Al-Absi, and G. Al-Naqeeb, "Thymoquinone rich fraction from Nigella sativa and thymoquinone are cytotoxic towards colon and leukemic carcinoma cell lines," Journal of Medicinal Plants Research, vol. 15, pp. 3359-3366, 2011.

[12] E. Halawani, "Antibacterial activity of thymoquinone and thymohydroquinone of Nigella sativa L. and their interaction with some antibiotics," Advances in Biological Research, vol. 3, pp. 148-152, 2009.

[13] A. Ragheb, A. Attia, W. S. Eldin, F. Elbarbry, S. Gazarin, and A. Shoker, "The protective effect of thymoquinone, an anti-oxidant and anti-inflammatory agent, against renal injury: a review," Saudi Journal of Kidney Diseases and Transplantation, vol. 20, no. 5, pp. 741-752, 2009.

[14] W. Tiyaboonchai, "Chitosan nanoparticles: a promising system for drug delivery," Naresuan University Journal, vol. 11, pp. 5166, 2003.

[15] M. V. Jothi, K. M. Bhat, P. K. Pratibha, and G. S. Bhat, "The evaluation of a biodegradable dental chip containing chlorhexidine in chitosan base as a targeted drug delivery in the management of chronic periodontitis in patients," Drug Development Research, vol. 70, no. 5, pp. 395-401, 2009.

[16] M. G. Newman, H. Takei, P. R. Klokkevold, and F. A. Carranza, Carranza'S Clinical Periodontology, Elsevier Health Sciences, Philadelphia, Pa, USA, 2011.

[17] U. Kalus, A. Pruss, J. Bystron et al., "Effect of Nigella sativa (Black Seed) on subjective feeling in patients with allergic diseases," Phytotherapy Research, vol. 17, no. 10, pp. 1209-1214, 2003.

[18] B. H. Ali and G. Blunden, "Pharmacological and toxicological properties of Nigella sativa," Phytotherapy Research, vol. 17, no. 4, pp. 299-305, 2003.

[19] M. A. Randhawa and M. S. Al-Ghamdi, "A review of the pharmacotherapeutic effects of Nigella sativa," Pakistan Journal of Medical Research, vol. 41, pp. 77-83, 2002.

[20] S. O. Arslan, E. Gelir, F. Armutcu et al., "The protective effect of thymoquinone on ethanol-induced acute gastric damage in the rat," Nutrition Research, vol. 25, no. 7, pp. 673-680, 2005.

[21] A. A. Mariod, R. M. Ibrahim, M. Ismail, and N. Ismail, "Antioxidant activity and phenolic content of phenolic rich fractions obtained from black cumin (Nigella sativa) seedcake," Food Chemistry, vol. 116, no. 1, pp. 306-312, 2009.

[22] T. Allahghadri, I. Rasooli, P. Owlia et al., "Antimicrobial property, antioxidant capacity, and cytotoxicity of essential oil from cumin produced in Iran," Journal of Food Science, vol. 75, no. 2, pp. H54-H61, 2010.

[23] J. R. Gonzales, L. Harnack, G. Schmitt-Corsitto et al., "A novel approach to the use of subgingival controlled-release chlorhexidine delivery in chronic periodontitis: a randomized clinical trial," Journal of Periodontology, vol. 82, pp. 1131-1139, 2011.

[24] D. C. Grisi, S. L. Salvador, L. C. Figueiredo, S. L. S. Souza, A. B. Novaes, and M. F. M. Grisi, "Effect of a controlled-release chlorhexidine chip on clinical and microbiological parameters of periodontal syndrome," Journal of Clinical Periodontology, vol. 29, no. 10, pp. 875-881, 2002. 

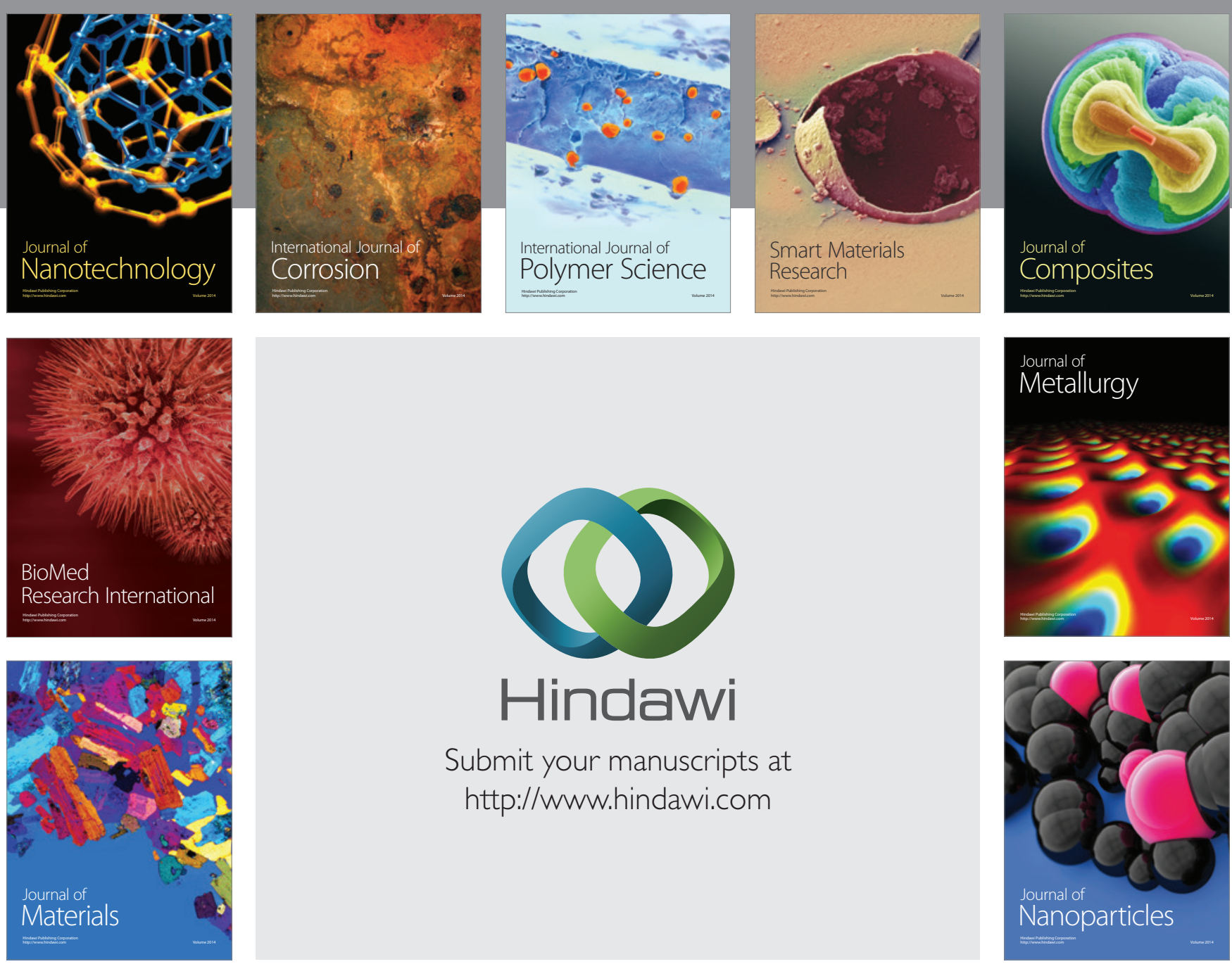

Submit your manuscripts at http://www.hindawi.com
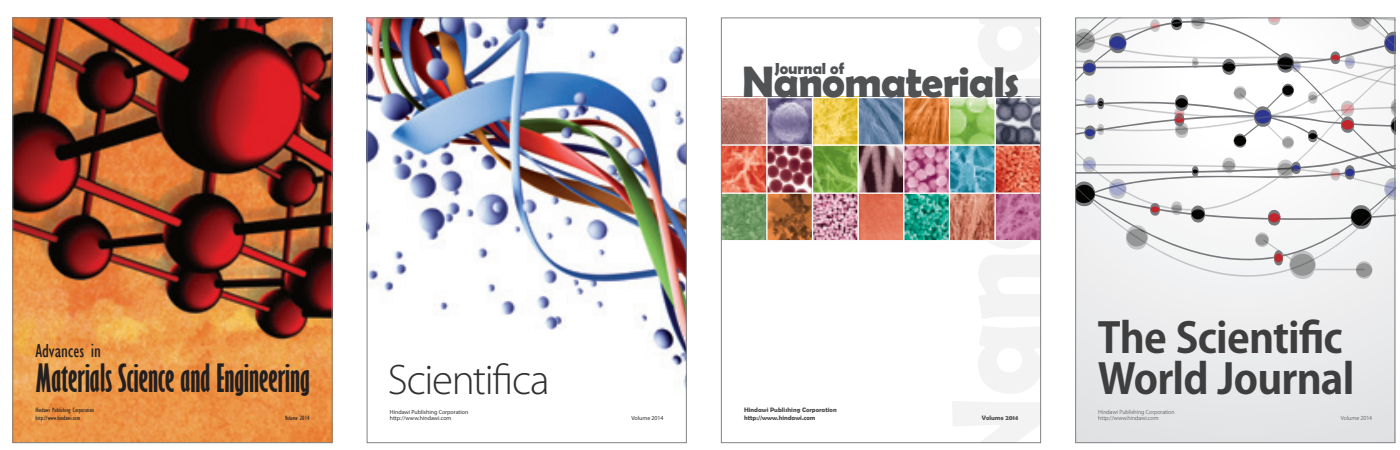

\section{The Scientific World Journal}
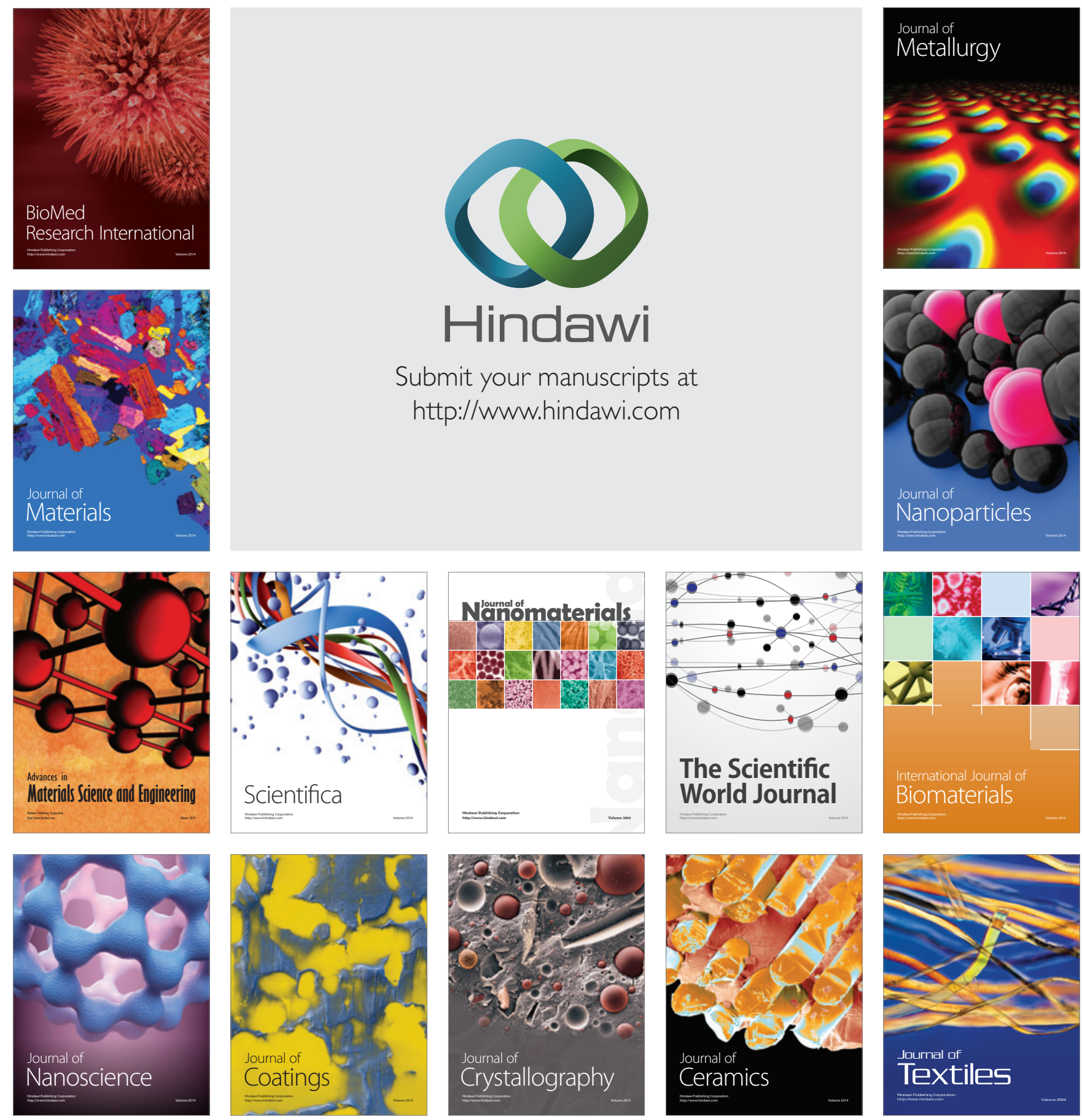